\title{
Pessoas em Situação de Rua no Brasil: Revisão Sistemática
}

\author{
Aline Amaral Sicari ${ }^{1}$ \\ ${ }^{1}$ Universidade Federal de Santa Catarina, SC, Brasil.
}

\author{
Andrea Vieira Zanella ${ }^{1}$ \\ ${ }^{1}$ Universidade Federal de Santa Catarina, SC, Brasil.
}

Resumo: A presença de pessoas que fazem das ruas sua moradia é uma realidade inconteste e vem se intensificando no Brasil. Realizou-se esta pesquisa com o objetivo de investigar o que foi produzido sobre pessoas nessa condição entre 2006 a 2016 no Portal de Periódicos Capes e no Banco de Teses \& Dissertações Capes. Foram analisados os resumos e as conclusões de 115 produções científicas brasileiras, sendo 27 artigos, 81 dissertações e sete teses. Constatou-se que no ano de 2014 houve maior produção científica sobre o tema (19,13\%) e que há uma concentração de pesquisas na área da Psicologia (26,13\%). As produções científicas apresentaram temas variados, sendo categorizadas por tópicos: singularidades e diversidades; razões e motivações para a vida nas ruas; os diferentes modos de viver e sobreviver em situação de rua; construção social da imagem; interlocuções entre saúde e assistência social; relação com a cidade e os processos de saída das ruas. Embora o interesse em investigar o fenômeno de pessoas que habitam as ruas venha se intensificando, indicamos a necessidade de novas pesquisas para que possam apontar indicadores para a construção de políticas públicas atenta aos direitos dessa população.

Palavras-chave: Pessoas em Situação de Rua, População em Situação de Rua, Revisão Sistemática.

\section{Homeless People in Brazil: A Systematic Review}

\begin{abstract}
The presence of people who make the streets their home is an incontestable reality and has been intensifying in Brazil. This research was carried out with the objective of investigating the academic production about this subject between 2006 and 2016. The research was done using the Capes Portal of Journals and Capes Bank of Theses and Dissertations. The abstracts and conclusions of 115 Brazilian scientific productions were analyzed, being twenty seven (27) articles, eighty one (81) dissertations and seven (7) theses. It was found that in 2014 there was a greater scientific production on the subject (19.13\%) and that there is a concentration of research in the area of Psychology (26.13\%). The scientific productions presented diverse themes, being categorized by topics: singularities and diversities; reasons and motivations for street life; the different ways of living and surviving in street situations; social construction of the image; interlocutions between health and social assistance; relationship with the city and the processes of leaving out the streets. Although the interest in investigating the phenomenon of people living on the streets is intensifying, we indicate the need for new research so that it can point out indicators for the construction of public policies focused on the rights of this population.
\end{abstract}

Keywords: Homeless People, Homeless Population, Systematic Review. 


\section{Personas en Situación de Calle en Brasil: Revisión Sistemática}

Resumen: La presencia de personas que hacen de las calles su vivienda es una realidad incontestable y viene intensificándose en Brasil. Se realizó este análisis con el objetivo de examinar las investigaciones sobre personas en esa condición, que fueron producidas entre 2006 y 2016 en el Portal de Periódicos Capes y en el Banco de Tesis \& Disertaciones Capes. Se analizaron los resúmenes y las conclusiones de 115 producciones científicas brasileñas, compuestas por veinte siete (27) artículos, ochenta y una (81) disertaciones y siete (7) tesis. Se constató que en el año 2014 hubo una producción científica más relevante sobre el tema $(19,13 \%)$, y que hay una concentración mayor de investigaciones en el área de la Psicología $(26,13 \%)$. Las producciones científicas presentaron temas variados, siendo categorizados por tópicos: singularidades y diversidades; razones y motivaciones para la vida en las calles; los distintos modos de vivir y sobrevivir en situación de calle; construcción social de la imagen; interlocuciones entre salud y asistencia social; la relación con la ciudad y los procesos de salida de las calles. Aunque el interés de estudiar el fenómeno de las personas que viven en las calles esté creciendo, señalamos la necesidad de nuevas investigaciones que puedan apuntar indicadores para la construcción de políticas públicas atentas a los derechos de esa población.

Palabras clave: Personas en Situación de Calle, Población en Situación de Calle, Revisión Sistemática.

\section{Introdução}

A presença de pessoas que fazem das ruas das metrópoles contemporâneas sua moradia é uma realidade inconteste. Na primeira metade do século XX esse fenômeno foi intensificado no Brasil com o êxodo rural e com o processo migratório, impulsionado pelo crescimento industrial (Brasil, 2009; CRP-MG, 2015). $\mathrm{O}$ panorama atual indica, entretanto, que a maioria das pessoas em situação de rua ${ }^{1}$ são provenientes de áreas urbanas, sendo o deslocamento do campo para a cidade não mais uma realidade frequente (Brasil, 2009).

$\mathrm{O}$ número de pessoas vivendo nas ruas vem se intensificando nos últimos anos e não se restringe às grandes metrópoles. Na Pesquisa Nacional da População em Situação de Rua, realizada entre agosto de 2007 e março de 2008 pelo Ministério do Desenvolvimento Social e Combate à Fome em 71 cidades do país (23 capitais e 48 municípios com mais de 300 mil habitantes), foram identificadas 31.922 pessoas em situação de rua maiores de 18 anos. Nessa condição foram consideradas tanto as pessoas que pernoitam em instituições como albergues e casas de passagem, como as que se localizam nas calçadas, praças, rodovias, lugares abandonados, becos e outros (Brasil, 2009).

A pesquisa traçou o perfil dessa população: predominam pessoas do sexo masculino (82\%), a maioria com idade entre 25 e 44 anos (53\%) e que se declaram pardas (39,1\%); $74 \%$ sabem ler e escrever; $70,9 \%$ exercem alguma atividade remunerada, como catador de material reciclável e flanelinha; $51,9 \%$ possuem algum familiar na cidade em que se encontram, mas 38,9\% não mantêm contato com seus parentes; $95,5 \%$ não são vinculados a nenhum movimento social e $24,8 \%$ não possuem nenhum documento de identificação (Brasil, 2009).

O estudou revelou que as razões que levam pessoas a viverem nas vias das cidades são variadas. A precarização das condições de vida é uma delas, porém reduzir a compreensão do fenômeno a questões eco-

\footnotetext{
${ }^{1}$ Para falar das pessoas que usam as ruas como espaço de circulação e permanência, em vez de utilizarmos o termo "morador de rua" optamos nesta pesquisa por "pessoa em situação de rua". O uso desta terminologia é apontado como o mais adequado, pois "pessoas" remete a humanidade, pluralidade e multiplicidade nas diferentes formas de existir, e "situação" como algo que é "transitório, em ininterrupta transformação, assim como ao se situar em um espaço particular, simbolizado pela rua" (Mattos, 2006, p. 225). Para Silva (2006), essa terminologia distancia-se de uma perspectiva simplista, reducionista e individualizante. Entretanto, Santos (2014) problematiza o uso do termo "situação de rua", pois acredita que este exclui o grupo populacional que tem uma relação duradoura, crônica com o habitar as ruas. Trata-se de uma problematização importante, pois de acordo com a pesquisa nacional, $30 \%$ das pessoas estão vivendo nas ruas a mais de cinco anos (Brasil, 2009).
} 
nômicas limita sua compreensão e reduz a complexidade que o conota. De acordo com a pesquisa nacional supracitada, os principais motivos relatados que levaram as pessoas a viver nas ruas é o uso abusivo de álcool e outras drogas (35,5\%), o desemprego (29,8\%) e conflitos familiares $(29,1 \%)$. A justificativa da ida e permanência nas ruas devido a uma escolha pessoal também aparece, não de forma frequente e expressiva, porém deve ser levada em consideração. Ao relatarem a escolha como a responsável pela vida nas ruas, justificam que a busca pela sensação de liberdade é a principal motivação (Brasil, 2009).

Considerando a importância de visibilizar melhor a realidade das pessoas em situação de rua, este estudo investiga o que tem sido produzido sobre essa temática no Brasil. Trata-se, pois, este estudo, de uma revisão sistemática do conhecimento científico produzido sobre essa população, realizado com o intuito de compreender as questões que vem sendo problematizadas e os enfoques adotados.

\section{Método}

Esta pesquisa é considerada como revisão sistemática ou revisão bibliográfica. De acordo com Ferreira (2002), neste tipo de pesquisa o objetivo é conhecer o que tem sido produzido em âmbito acadêmico sobre um tema específico e discutir essa produção, considerando o tempo, o lugar e o contexto. A metodologia deste tipo de pesquisa possui caráter "inventariante e descritivo das produções acadêmicas e científicas sobre o tema" (Ferreira, 2002, p. 258). Para Zoltowski, Costa, Texeira e Koller (2014), a revisão sistemática se define por uma estratégia de busca das produções científicas, uma análise crítica e uma síntese do que foi encontrado.

Para identificar a produção científica sobre pessoas em situação de rua no Brasil, escolhemos como fonte de busca o Portal da Coordenação de Aperfeiçoamento de Pessoal de Nível Superior (Capes²) e o Banco de Teses \& Dissertações ${ }^{3}$ da Capes. O levantamento das produções científicas em ambos os portais foi realizado em Janeiro de 2017, delimitando a busca entre o período de 2006 a 2016, sem filtrar o tipo de produção científica. Justo (2008) afirma que até os anos de 1970 quase não existia bibliografia sobre pessoas que viviam nas ruas, e que a partir de 1990 tornou-se mais frequentes estudos sobre esta temática. Mendes, Ronzani e Paiva ${ }^{4}$ (no prelo) realizaram uma busca entre os anos de 1990 a 2015 e identificaram o crescimento de artigos sobre a temática a partir do ano de 2006. Assim, justifica-se a delimitação do levantamento a partir de 2006, considerando o aumento dos estudos sobre a temática.

No Portal Capes utilizamos para a busca a associação do descritor ${ }^{5}$ "pessoas em situação de rua" e a palavra-chave "morador de rua", através do operador booleano OU, o que resultou em 42 produções científicas. No Banco de Teses \& Dissertações não é possível realizar associação de termos chave e nem delimitar o ano das produções. Dessa forma, a primeira busca nesse portal foi feita apenas com o descritor "pessoas em situação de rua”, e resultou em 86 produções científicas. A segunda busca foi realizada com a palavra-chave "morador de rua", resultando em 64 estudos.

No total, foram encontradas 192 produções científicas (artigos, teses e dissertações). Foram definidos como critérios de exclusão a duplicidade ou triplicidade de arquivos, pesquisas realizadas fora do período delimitado dos últimos dez anos, estudo realizado fora do Brasil, arquivos indisponíveis online e com temas não referentes a pessoas em situação de rua. Como exemplo de produção científica excluída está o estudo de Garcia, Calderón e Ferreira (2012) sobre populações caninas em áreas urbanas. Adotando os critérios de exclusão, a leitura dos resumos resultou na seleção de 115 produções científicas, sendo 27 artigos, 81 dissertações e sete teses.

Considerando a heterogeneidade e a diversidade dos resumos acadêmicos, realizou-se também a leitura das considerações finais/conclusões dos trabalhos. Destacamos que algumas das 115 produções foram lidas na íntegra, em virtude de trazerem uma discussão mais aprofundada do problema em foco ou pelo fato das informações apresentadas nos resumos e considerações finais não serem suficientes para a compreensão.

\footnotetext{
${ }^{2}$ Disponível em: http://www.periodicos.capes.gov.br/

${ }^{3}$ Disponível em: http://bancodeteses.capes.gov.br/banco-teses/\#/

${ }^{4} \mathrm{O}$ artigo de Mendes, Ronzani e Paiva está no prelo. O acesso a este artigo ocorreu através do contato com uma das autoras. Em decorrência, não está incluído quantitativamente nas análises.

${ }^{5}$ Descritores são termos padronizados, definidos por especialistas. Palavras-chave são termos simples, definidos pelos autores. A escolha do descritor foi definida através do portal Descritores em Ciências da Saúde (http://decs.bvs.br/).
} 
Foram consideradas nas análises informações referentes a área, vinculação institucional, ano, metodologia utilizada e resultados. Buscou-se articular as informações para obter uma compreensão crítica do que já foi pesquisado (Oliveira, Trancoso, Bastos \& Canuto, 2015). Para apresentação das análises realizadas, foram organizadas as seguintes unidades temáticas: As singularidades e diversidades das pessoas em situação de rua; Razões e motivações para a vida nas ruas; $\mathrm{O}$ s diferentes modos de viver e sobreviver em situação de rua; A construção social da imagem da pessoa em situação de rua; Interlocuções entre saúde e assistência social; A relação com a cidade; e Os processos de saída das ruas.

\section{Discussão}

Das 81 dissertações e sete teses encontradas, 26,13\% foram realizadas em Programas de Pós-Graduação em Psicologia; 7,95\% em Programas de Pós-Graduação em Saúde Pública/Saúde Coletiva e 7,95\% em Programas de Pós-Graduação em Serviço Social. Há também estudos em outras áreas, como: comunicação, linguística, educação, arquitetura e urbanismo, administração, engenharia, geografia, políticas públicas, ciências da religião, antropologia, ciências sociais, gerontologia, enfermagem, direito e artes. Em relação aos 27 artigos científicos encontrados, destacaram-se publicações na revista "Interface: Comunicação, Saúde e Educação”, de caráter interdisciplinar, contabilizando um total de $18,51 \%$ dos estudos identificados.

Das teses e dissertações analisadas, houve relativa prevalência de vínculo institucional com universidades do estado de São Paulo, sendo 14,77\% estudos da Universidade do Estado de São Paulo (USP) e 7,95\% da Pontifícia Universidade Católica de São Paulo (PUC-SP). A Universidade de Brasília (UnB) e a Universidade Federal do Rio Grande do Sul (UFRGS) também apresentaram $6,81 \%$ e 5,68\% respectivamente. Identificamos estudos de diversas outras instituições de ensino superior, como: Universidade Federal do Ceará (UFC), Pontifícia Universidade Católica do Rio de Janeiro (PUC-Rio), Universidade Estadual do Ceará (UECE), Universidade Federal de Santa Catarina (UFSC), Universidade Federal de Juiz de Fora (UFJF), Universidade Federal da Bahia (UFBA), Universidade Católica de Salvador, Universidade Metodista de São Paulo, dentre outras.

Em relação ao ano das produções científicas, identificamos oscilação significativa entre 2006 e
2016, sendo os anos de 2013, 2014 e 2015 com maior número de publicações. No ano de 2014 houve a maior quantidade de produções científicas, totalizando 19,13\%. O decréscimo dessas produções em 2016 deve ser relativizado, pois a busca bibliográfica foi realizada em janeiro de 2017, e é possível que algumas produções de 2016 não tivessem ainda sido publicadas e/ou inseridas nos portais de pesquisas.

As produções analisadas apresentaram variadas escolhas metodológicas: etnografia, cartografia, pesquisa-intervenção, observação participante, história de vida, grupo focal, questionários, genealogia, arqueologia, documental e outras. A maioria das produções $(38,26 \%)$ utilizou técnica de entrevistas para produção e coleta das informações, sendo presente tanto o uso exclusivo de entrevistas quanto o uso associado a outra técnica. O método etnográfico e a pesquisa documental foram citados cada um em 18,26\% dos estudos.

Dentre as produções analisadas, nenhuma apresentou um levantamento bibliográfico exclusivo sobre "pessoas em situação de rua" ou "morador de rua". Foram identificadas revisões de literatura sobre o tema (Borysow, \& Furtado, 2013; Cândido, 2006; Quintão, 2012), mas sempre vinculando a pessoa em situação de rua a algum outro descritor, como alguma vulnerabilidade social ou característica específica.

Mendes et al. (no prelo) realizaram uma revisão sistemática utilizando o descritor "drogas" cruzado com outras palavras-chave, sendo uma delas "população em situação de rua". O objetivo foi compreender a forma como as condições de vulnerabilidade social, pobreza, estigma e raça são relacionadas às pessoas em situação de rua usuárias de drogas. Os autores compararam as produções acadêmicas estadunidenses e latino-americanas, e identificaram que nas primeiras existe o foco nas análises de riscos e a categorização quantitativa das informações, enquanto nas segundas o foco é a contextualização das condições sociais das pessoas em situação de rua e as razões que levaram a estar na referida situação. Borysow e Furtado (2013) realizaram uma revisão narrativa sobre a temática de pessoas em situação de rua que possuem o diagnóstico de algum tipo de transtorno mental grave. Cândido (2006) utilizou a metodologia bibliográfica para conceituar a ação das pastorais católicas com as pessoas em situação de rua na cidade de São Paulo, e Quintão (2012) buscou compreender a cidade a partir das pessoas que habitam as ruas. 


\section{As singularidades e diversidades das pessoas em situação de rua}

Diversos estudos tiveram como sujeitos de pesquisa subgrupos e/ou características dessa população: idosas, mulheres, crianças e jovens, com saúde mental prejudicada ou uso de substâncias psicoativas, a relação com a religiosidade, relações com o trabalho, egressos do sistema prisional e a relação dessas pessoas com animais de estimação. A seguir, apresentaremos o que estes estudos evidenciam em relação a cada subgrupo investigado.

Bueno (2013), ao pesquisar pessoas idosas em situação de rua, identificou o uso recorrente de álcool, ausência de renda financeira, autodeclaração de algum tipo de doença e a afirmação de não se perceberem envelhecendo, mas que se sentem velhos antes dos 60 anos. $\mathrm{O}$ autor identificou que o processo de envelhecimento dessas pessoas acontece de forma rápida, devido ao meio social e às condições precárias de vida. Saldanha (2014) constatou que os idosos em situação de rua possuem maiores dificuldades de inserção no mercado de trabalho, tanto formal quanto informal. Brêtas, Marcolan, Rosa, Fernandes e Raizer (2010) concluem que a rua é um ambiente geralmente desfavorável ao envelhecimento; apontam que ao viver na rua condições mínimas de bem-estar e de saúde não podem ser atingidas, desencadeando prejuízos na autoestima e no autocuidado; destacam ainda a falta de eficiência de políticas públicas que possibilitem a saída dessa condição.

Com relação às pesquisas que buscaram compreender as especificidades e os modos de vida de mulheres, Rodrigues (2009) identificou que o número de mulheres nas ruas é de duas para cada dez homens, com faixa etária diversa. Rosa e Brêtas (2015) apontam que os fatores predominantes que ocasionaram a ida de mulheres para as ruas estão a violência doméstica, dificuldade financeira e o rompimento com vínculos sociais. Já nas ruas, essas mulheres também enfrentam a violência do tráfico, da repressão policial e estatal, da disputa por território e a violência de gênero, além das dificuldades em obter privacidade, dados também apontados por Alves (2013). As pesquisas destacam que a rua se configura como um espaço masculino, que dá continuidade ao modelo patriarcal. Mas ressaltam que as mulheres em situação de rua, mesmo com fortes opressões, conquistam e ocupam seu espaço, sendo protagonistas de suas próprias vidas (Alves, 2013; Rodrigues, 2009; Rosa, \&
Brêtas, 2015). Pesquisadores destacam que não há, nas políticas públicas brasileiras, ações direcionadas às especificidades das mulheres nas ruas, apontando a necessidade de estratégias singulares e a construção intersetorial de cuidado entre políticas públicas de mulheres e de pessoas em situação de rua (Alves, 2013; Rosa, \& Brêtas, 2015; Santos, 2014).

Com relação a crianças e jovens em situação de rua, Moura, Silva e Noto (2009) afirmam que, assim como para a população adulta em situação de rua, para as crianças e jovens, as razões que ocasionam esse processo são múltiplas, evidenciando a fragilidade dos vínculos familiares. Para as autoras, o uso abusivo de drogas está intrinsecamente ligado à vulnerabilidade do âmbito familiar e funciona como um propulsor para a ida às ruas. Cofani (2012) evidenciou que jovens em situação de rua iniciam o consumo de álcool mais cedo que jovens de outros grupos sociais. Moura et al. (2009) e Matias (2013) identificaram a manifestação do sentimento de ambivalência e polarização dessa população em relação à rua. Afirmam que no primeiro momento a rua se configura como espaço de liberdade e independência, mas logo em seguida, surgem as dificuldades de sobrevivência básica, configurando-se como espaço de privações.

Outro recorte frequente nas pesquisas encontradas é a intersecção de pessoas em situação de rua e usuárias de álcool e outras drogas, mais especificadamente o uso abusivo de crack. Alles (2010) e Morera (2013) identificaram que a situação de rua está associada, predominantemente, ao uso de crack, condição social atrelada a fatores como: ausência de regras e normas sociais; núcleo familiar violento em que há pouco afeto e diálogo; baixa renda e desemprego. Varanda (2009) e Tondin, Barros Neta e Passos (2013) afirmam que o uso de substâncias psicoativas se configura como uma alternativa para resistir às condições sociais adversas, sobreviver e minimizar o sofrimento físico e psíquico. Campos (2012) aponta que o álcool exerce um duplo papel na vida das pessoas em situação de rua: ora tem a função de ser confortante e consolador, ora é o motivo e a causa da condição de rua. Gomes e Adorno (2011) acompanharam a trajetória de pessoas em situação de rua e apontam para a ineficácia de políticas públicas repressivas e as potencialidades do cuidado respaldado pela perspectiva da redução de danos.

Ao estudar sobre pessoas em situação de rua e a relação dessas com a religiosidade, Guimarães (2010) identificou a religiosidade como uma via de subjetiva- 
ção e um modo de darem sentido à existência. Honorato (2014) evidencia a prática subversiva de adesão religiosa, como o comportamento de empatia pela religião de algum grupo que realiza doações à população de rua. Para o pesquisador, esta subversão se configura como uma forma de resistência aos discursos religiosos impostos em troca de benefícios sociais. Galvani (2015) afirma que as ações de doação dos grupos religiosos geram tensões e incômodos, pois a prática de "ter que rezar" antes de receber o alimento foi percebida, por alguns interlocutores, como "uma forma de humilhação" (p. 141). Candido (2006) afirma que as ações realizadas por grupos religiosos promovem intervenção sócio-política ampliando o cuidado e a humanização, porém evidenciam a ineficácia de políticas públicas sociais.

Pesquisas apontam que é comum para pessoas em situação de rua o trabalho de vigias de carros, catadores de material reciclável e a mendicância como fonte de recursos (Alles, 2010; Lacerda, 2012). São quase inexistentes oportunidades de trabalho formal devido ao preconceito e ausência de endereço fixo, mesmo que a pessoa tenha competência e qualificação requeridas (Alles, 2010; Oliveira, 2015). Entretanto, algumas pessoas que desenvolvem as atividades acima mencionadas não reconhecem suas estratégias de sobrevivência financeira como trabalho (Lacerda, 2012). Carvalho (2015) aponta para a necessidade de conferir o status de trabalho a algumas atividades informais de sobrevivência como fundamental tanto socialmente como subjetivamente.

Büll (2010) identificou que o conceito de trabalho para pessoas em situação de rua assume um papel constitutivo da subjetividade, sendo na maioria das vezes considerado como sinônimo de honestidade e pertença social, não sendo exercido com o objetivo de acúmulo de dinheiro ou bens, mas direcionado à sobrevivência nas ruas. Carvalho (2015) afirma que as pessoas em situação de rua aceitam, na maioria das vezes, qualquer tipo de trabalho, pois se encontram em situação de vulnerabilidade e não conseguem exigir nenhuma garantia e/ou direito trabalhista. Langa (2012) identificou que a inserção no trabalho informal e/ou ilegal é algo que acompanha a trajetória de vida dessas pessoas, mesmo antes de viverem nas ruas. Farias (2007) evidencia a necessidade de políticas públicas que possam fomentar a inserção em atividades produtivas, a fim de promover geração de renda e uma vida digna.

Um estudo investigou a condição de vida do egresso do sistema prisional em situação de rua. Karam
(2015) evidencia a ineficácia da metodologia de ressocialização dos presídios brasileiros, a qual serve para embrutecer o sentenciado, enfraquecer os laços sociais e familiares, além de não oferecer condições objetivas de uma vida fora das prisões; o processo de reinserção social culmina na situação de rua.

Uma produção científica investigou a relação de pessoas em situação de rua com seus animais de estimação. Cunha (2015) identificou que essa relação é de companheirismo e proteção, pois os animas auxiliam na vigilância e nos riscos de estar em situação de rua. A autora percebeu que há dificuldade dessas pessoas em frequentarem espaços que seus cães não são permitidos, como exemplo instituições de abrigamento. Assim, ressalta a necessidade das políticas públicas regulamentarem o acesso das pessoas em situação de rua com seus animais em serviços socioassistenciais.

\section{Razões e motivações para a vida nas ruas}

Várias pesquisas identificam como motivos e razões que levaram pessoas a viverem nas ruas o uso abusivo de álcool e outras drogas, o rompimento de vínculos e os conflitos familiares (incluindo separação conjugal), o desemprego e o prazer da liberdade que é vivenciado na rua (Abreu, 2013; Aguiar, 2014; Alcantara, Abreu, \& Farias, 2015; Campos, 2012; Costa, Mesquita, \& Campos, 2015; Esquinca, 2013; Saldanha, 2014). Campos (2012) aponta que as questões multifatoriais que ocasionam a situação de rua podem acontecer de forma gradual e processual, culminando na ocupação da rua de forma permanente.

Para Argiles (2012) e Silva (2015), o fenômeno de pessoas em situação de rua vem aumentando devido à precarização das relações de trabalho, o desemprego e as transformações econômicas. Costa e Richetti (2011) e Souza, Silva e Caricari (2007) evidenciam que esse fenômeno está ligado ao processo de globalização, em que a exclusão social se intensifica. Tondin et al. (2013) explicam que os sentimentos de exclusão e sofrimento, produto dos processos econômicos e políticos baseados na injustiça social, culminam na situação de rua. Para Silva (2006), essa condição se configura como uma síntese de determinações sociais fortemente marcadas pelo sistema capitalista.

Saldanha (2014) identificou que algumas pessoas estão em situação de rua devido a ameaças de milícias ou traficantes que as obrigaram a sair de suas casas, impedindo o retorno para os locais que moravam. Para Langa (2012), a situação de rua pode ser para 
algumas pessoas a única alternativa diante do percurso de exclusão e vulnerabilidade social presentes em várias etapas da vida. Saldanha (2014) identificou que uma parcela das pessoas em situação de rua apresenta histórico de migração de uma cidade para outra em busca de oportunidades e, na ausência dessas, acabam permanecendo na nova localização, com poucos recursos financeiros e sociais, o que ocasiona a situação de rua.

Mesmo tendo um número significativo de pesquisas que apontam para os múltiplos fatores que ocasionam a vida nas ruas e que consideram o contexto social, histórico e político, algumas destacam outros aspectos. Esmeraldo Filho (2010) identificou que algumas pessoas associam a atual condição como algo de sua responsabilidade, desconsiderando os processos sociais e econômicos. Silveira (2016) identificou como justificativas predominantes para a situação de rua a questão socioeconômica, a saúde mental prejudicada e o desejo de estar na rua. Rodrigues (2015) relaciona a ideia da escolha individual de estar nas ruas com o que chama de "mito da escolha masoquista" (p. 7), um processo vinculado à ideologia neoliberal de transformação da ideia de necessidade para a de escolha da pessoa em situação de rua que promove a "transformação da necessidade e do sofrimento em escolha [...] no qual a miséria não aparece como adereço das indigências fabricadas por estruturas societárias - a sociedade transfere assim, a culpa e a responsabilidade para o indivíduo" (p. 78). Nessa lógica, a pessoa em situação de rua é considerada não só culpada por sua própria condição, mas também responsável por problemas coletivos, como a violência, o tráfico, a degradação urbana e outras adversidades (Rodrigues, 2015).

\section{Os diferentes modos de viver e sobreviver em situação de rua}

$\mathrm{O}$ interesse em conhecer os diferentes modos de viver em situação de rua esteve presente em diversas investigações. Alles (2010) afirma que, dentre as dificuldades encontradas em estar em situação de rua, as relacionadas à sobrevivência são as mais mencionadas. $\mathrm{O}$ autor afirma que estar em situação de rua gera sofrimento, pois vivenciam desde dificuldades de sobrevivência física (fome e frio) quanto relacionais: na maioria das vezes, sofrem preconceito, estigmatização, desrespeito e violência policial, questões também apontadas por Al Alam (2014), Aguiar (2014) e Abreu (2013).
Campos (2012) identifica que quase sempre a ideia de viver nas ruas remete a perdas, entretanto, chama a atenção para os aprendizados na rua, um local de elaboração de estratégias de enfrentamento e superação de dificuldades. Oliveira (2012) também aponta que durante muito tempo a rua foi considerada exclusivamente como um espaço de miséria e violência, e indica a necessidade de superar esta perspectiva e ampliar a concepção da rua, compreendendo-a como espaço da "capacidade humana da transformação, pois nela se constroem laços afetivos como amizades e amores, novos rearranjos e experiências na cidade" (p. 141). Para Moura Junior (2012), a rua também é espaço para construção de vínculos e demonstração de afetividade e liberdade.

Castiglioni (2012) ressalta a potência que permeia o processo de rualização, evidenciando que mesmo com as condições precárias e desfavoráveis, essas pessoas estão ativas no processo de construção de outros modos de viver, estando abertas a novas vinculações sociais e a processos de criação. Mendonça (2006) afirma que as pessoas em situação de rua produzem subjetivamente sentidos múltiplos sobre si, sobre o outro e sobre a vivência de rua. Silva (2014) compreende que essas pessoas produzem modos de existência atravessados pelos processos de desfiliação social: ruptura de vínculos sociais, distanciamento do mercado de trabalho e violações de direitos. Para a autora, elas são objetivadas e subjetivadas por processos marginalizantes que circunscrevem suas marcas nos corpos, fomentando a resistência frente ao controle de suas vidas.

Moura Junior (2012) identificou que a sociedade ainda atribui um caráter depreciativo às pessoas em situação de rua, ocasionando práticas discriminatórias e opressoras, negando as potencialidades das diferentes formas de existir. Mas destaca que elas resistem de algum modo, significando a rua como espaço de "potencialidades e de fortalecimento de vínculos, sendo atrativa para pessoas que, mesmo possuindo um domicílio, vivem em um terreno árido de esperança $\mathrm{e}$ repleto de opressão" (Moura Junior, 2012, p. 135).

Félix-Silva, Sales e Soares (2016) apontam as diferentes configurações da rua para cada pessoa que nela vive. Para alguns, a rua é um lugar de passagem, em que o medo e a dúvida acompanha todo o processo; para outros, é sinônimo de um lugar acolhedor, que abriga e aconchega quem se encontra sem ter para onde ir e quem decide ficar. Os autores destacam que viver em situação de rua pode se configurar como um modo de desenvolver a arte de viver mesmo em con- 
dições extremas de vulnerabilidade e precariedade social: a "arte de fazer arte como resistência política ativa, onde a vivência na rua se torna um vetor de corte" (p. 48), e que se configura como um dispositivo de produção de saúde mental desses sujeitos.

Langa (2012) identificou uma multiplicidade de modos de viver nas ruas: para algumas pessoas, é "espaço de riscos e perigos" (p. 162), de insegurança; para outras pessoas, a rua se configura "como um local de liberdade para viver" (p. 163), não mais desejam a moradia domiciliar, pois desfrutam da liberdade proporcionada para aqueles que obedecem os códigos da rua e manejam situações perigosas; em contraposição, para outras pessoas a rua é "a última alternativa, em um processo de vulnerabilidade" (p. 163): não constroem laços afetivos e sociais na rua e não possuem confiança e segurança.

A heterogeneidade como uma característica principal tanto da população em situação de rua como de suas maneiras de viver é afirmada em diversas pesquisas (Alcantara et al., 2015; Félix-Silva et al., 2016; Galvani, 2008; Lacerda, 2012; Langa, 2012; Mattos, 2006; Rodrigues, 2009; Rosa, Secco, \& Brêtas, 2006; Serrano, 2013). Lacerda (2012) aponta que a generalização de que a maioria vive sozinha, sem vínculos afetivos, não é aceitável. Lemões (2014) evidencia que nem sempre os vínculos familiares estão efetivamente rompidos, e que mesmo sofrendo políticas higienistas e de exclusão das cidades, as pessoas em situação de rua desenvolvem estratégias e vínculos sociais como forma de permanecerem nos espaços. Galvani (2008) afirma que essa heterogeneidade é premissa fundamental para a compreensão dos modos de vida dessas pessoas, identificando movimentos de resistência e fortalecimento dos modos de viver nas ruas, com a construção de redes sociais e o envolvimento com ações de cultura, arte, religião e movimentos sociais. Os modos de existir e a forma como vivem estão intrinsecamente relacionadas às práticas sociais estabelecidas no contexto da rua, sendo essas múltiplas, variáveis e diversas.

\section{A construção social da imagem da pessoa em situação de rua}

Oliveira (2012) afirma que o modo como a sociedade compreende as pessoas em situação de rua foi sendo transformado ao longo do tempo. Essas pessoas eram compreendidas como migrantes e desempregadas; no início dos anos 2000, começa-se a compreende-las a partir da exclusão e vulnerabilidade, o que fomentou o desenvolvimento de políticas públicas e intensificou o preconceito social. Serrano (2013) identifica uma rotulação da pessoa em situação de rua como doente, e afirma que a construção desse estereotipo está presente tanto nos estudos acadêmicos quanto nas práticas institucionais que exercem um controle sobre o corpo e a vida dessas pessoas, através da medicalização e das normas do cuidado em saúde e assistencial. Costa (2007) evidencia que a sociedade desloca a ideia de que a pessoa em situação de rua está em sofrimento para a concepção de que esta pessoa oferece algum tipo de risco e ameaça. Assim, demonstra que ocorrem mudanças com relação ao modo de se relacionar com estas pessoas, apontando uma progressiva racionalização que deixou de lado a solidariedade que existia anteriormente (Costa, 2010).

Lacerda (2012) e Sambu (2014) identificaram que existe na população em situação de rua os sentimentos de autorejeição e baixa autoestima devido às práticas estigmatizantes e de discriminação que vivenciam. Alles (2010) evidencia que essas pessoas enfrentam dificuldades para se relacionar com outras pessoas que não vivem nas ruas, precisando sempre se reafirmar como seres humanos e cidadãs para obter acesso a direitos, diminuir o preconceito e a estigmatização social. Em contrapartida, o autor destaca que os olhares a elas destinados são múltiplos, ora positivos e ora negativos. Alguns destilam ódio e preconceito, tratando-as com hostilidade e desconfiança, mas outros as beneficiam e auxiliam em diferentes aspectos, principalmente na alimentação (Alles, 2010).

Abib (2014) afirma que a sociedade tem construído a imagem das pessoas em situação de rua como um anormal contemporâneo, o que tem gerado formas de criminaliza-las. Abib (2014) e Costa (2009) chamam a atenção para o grande investimento midiático em reproduzir o estereotipo dessas pessoas, sempre relacionando-as à criminalidade, loucura e pobreza, semeando o medo e a insegurança social. Essa forma de pensamento viabiliza políticas públicas de limpeza urbana e de exclusão, além de uma transposição dos jogos de poder: se antes a pessoa em situação de rua e as usuárias de drogas eram responsabilidade exclusiva da justiça, com esses outros discursos essas pessoas passam a ser de responsabilidade também da área da saúde, deslocando a questão do poder judicial para o poder psiquiátrico e sua lógica normatizadora (Abib, 2014).

Almeida Junior (2014) analisou discursos jornalísticos de duas revistas, uma de grande circulação 
nacional e uma revista alternativa. Identificou que ambas reproduzem estereótipos em relação às pessoas em situação de rua, ou seja, constroem a imagem através de características gerais. Silva e Hüning (2015), ao analisar textos de jornais e outros documentos, identificaram a associação da pessoa em situação de rua a uma pessoa inevitavelmente criminosa. Frazão (2010) afirma que quando a imprensa jornalística divulga notícias sobre pessoas em situação de rua, o discurso é colado à seção policial com enunciados relacionando essas pessoas como suspeitos de crimes. Quando a notícia não é vinculada à criminalidade, está vinculada à degradação do espaço público (Frazão, 2010). Assim, o autor identificou que a construção da imagem da pessoa em situação de rua no discurso jornalístico é sempre referenciada a questões negativas, contribuindo para a manutenção de uma visão hegemônica excludente. Resende (2016) acrescenta que a representação midiática sobre a violação de direitos e a violência contra pessoas em situação de rua no jornalismo brasileiro é desprezada.

Em contrapartida, Alles (2010), ao analisar as publicações de um jornal feito por e sobre pessoas em situação de rua, identificou que em diferentes edições a representação social do que é um morador de rua é variável, sendo em algumas expostas suas vulnerabilidades e necessidades e em outras as potencialidades e a força de luta para melhorar suas condições de vida. Costa (2010) afirma que os meios de comunicação produzidos por pessoas em situação de rua são importantes para levar o tema da vida nas ruas para a sociedade, funcionando também como um instrumento de reivindicação de direitos sociais e humanos. Para Santos (2013), a produção do jornal por pessoas efetivamente em situação de rua, ou seja, em risco social, tem um peso material e simbólico contra-hegemônico. Ferreira (2007) afirma que o envolvimento dessas pessoas com a construção coletiva de jornais de rua configura uma via transformadora do modo de se relacionar com o espaço público e com a condição de situação de rua, bem como de regaste de autonomia.

\section{Interlocuções entre saúde e assistência social}

Para Borysow e Furtado (2013), é comum os serviços da assistência social assumirem a função de articuladores de rede quando se refere a pessoas em situação de rua. Para Lisboa (2013), a falta de articulação entre a saúde e a assistência social, principalmente em relação a questões referentes ao sofrimento psíquico no território, promove uma cronificação e institucionalização da pessoa. Borysow e Furtado (2013) e Montiel et al. (2015) evidenciam a necessidade do desenvolvimento e fortalecimento de uma rede intersetorial de cuidado à pessoa em situação de rua. Borysow e Furtado (2013) e Montiel et al. (2015) identificaram que os serviços de saúde mental ainda apresentam dificuldades em realizar atendimentos no local em que as pessoas em situação de rua vivem, bem como vincula-las às instituições devido às dificuldades em adaptarem e flexibilizarem suas estratégias de atuação.

Em relação aos serviços de assistência social, Costa et al. (2015) evidenciam a existência de uma linha tênue que diferencia a atividade profissional do campo da assistência social de atividades motivadas pela caridade. Costa (2007) relata que nos anos de 1970 e 1980, os primeiros grupos a oferecerem cuidado às pessoas vivendo nas ruas foram os religiosos. Posteriormente, nos anos de 1990, a atenção para as pessoas em situação de rua passou a fazer parte das ações institucionais e interventivas do estado, iniciando as parcerias públicas-privadas (Costa, 2007). Para Souza (2015), as políticas de assistência social atuais ainda estão fundadas no paradigma assistencialista e filantrópico, o que impede uma transformação das questões estruturais, mantendo o status quo e produzindo modos de subjetivação alienados. Souza et al. (2007) e Costa et al. (2015) apontam como necessária uma transição na prática das ações assistencialistas para ações que fomentem a emancipação e inclusão social, evidenciando a importância do desenvolvimento de ações intersetoriais.

Reis (2014) aponta o Centro de Referência Especializado para População em Situação de Rua (Centro POP), que está inserido no Sistema Único de Assistência Social (SUAS), como um espaço potente que possibilita a criação de vínculos e atende as necessidades imediatas da população. Porém, destaca a necessidade de que esse serviço assistencial ofereça um "processo de consciência desses sujeitos, despertando-lhes sua capacidade de agentes históricos que respondem a determinadas necessidades, sendo capazes de ressignificá-las" (Reis, 2014, p. 144). Argiles (2012) identificou nas pessoas em situação de rua investigadas o sentimento de crença e confiança nos serviços prestados pelo Centro POP, porém destaca que esse serviço precisa desenvolver mais ações que atendam as pessoas em suas singularidades, garantindo-lhes direitos básicos e acesso à rede intersetorial. 
Ferreira, Rozendo e Melo (2016) identificaram a necessidade de abertura de diálogo entre a saúde mental e a atenção básica. Para os autores, é necessário que a atenção especializada em saúde mental descentralize suas ações e que a atenção básica se aproxime de pessoas em situação de rua para oferecer um cuidado mais adequado. Macerata, Soares e Ramos (2014) traçam uma linha de conexão entre as ações da atenção básica com a população em situação de rua, pontuando ser imprescindível a compreensão de território como espaço de surgimento e emergência de vidas e de política.

Al Alam (2014) afirma serem necessárias discussões sobre as reais necessidades desse grupo populacional, de modo a reconhecer suas vulnerabilidades e desenvolver ações de atenção à saúde e assistência social com qualidade, comprometidas com direitos humanos e sociais e desenvolvidas no território. Matraga, Araújo-Jorge eWimmer (2014), Londero, Ceccim e Bilibio (2014) e Silveira (2016) afirmam que para ofertar um cuidado contextualizado e humanizado às pessoas em situação de rua, são necessárias ações que preparem os profissionais em educação permanente, educação popular e arte.

Para Miranda (2014), a situação de rua é a forma mais nítida da desigualdade social, o que invoca a necessidade da construção de ações afirmativas na garantia de direitos, dentre eles o direito à saúde. Miranda (2014) e Carvalho (2014) identificam que existem iniquidades que impedem o acesso direto e livre dessa população aos serviços de saúde, indicando serem necessárias ações e estratégias que modifiquem essa situação. Esmeraldo Filho (2010) evidencia que são diversos os problemas de saúde que acometem esta população, sendo a maioria relacionado ao abuso de substâncias psicoativas e a violência.

Para Nogueira (2008), pessoas em situação de rua têm como principal meio de acesso à saúde os serviços que atendem urgências e emergências. Rosa et al. (2006) identificaram que os profissionais de saúde ainda não conseguem compreender a pessoa em situação de rua em relação ao processo de saúde-doença-cuidado. Ferreira (2015) aponta que há uma inadequação organizacional em relação aos profissionais da rede de saúde: constata que ainda há preconceitos e estigmas em relação às pessoas em situação de rua, o que demonstra ser necessário engajamento da gestão a fim de ofertar uma política de cuidado que compreenda essas pessoas como cidadãos de direitos.

Com relação à oferta de cuidado no território, encontramos um número significativo de pesquisas que analisam o dispositivo de saúde da atenção básica direcionado às pessoas em situação de rua, o Consultório na Rua (CnaR) (Abib, 2014; Abreu, 2013; Albuquerque, 2014; Ferreira, 2015; Ferreira et al., 2016; Londero et al., 2014; Tondin et al., 2013; Lima, 2013). Albuquerque (2014) define o CnaR como um dispositivo analisador do processo da reforma psiquiátrica brasileira, pois fomenta a responsabilização de cuidar em liberdade e no território, tensionando os desafios da não institucionalização. Ferreira et al. (2016) notaram que o CnaR é visto como um serviço de saúde, mas também como um suporte social e de afeto, que possibilita transformações e potencialidades nos modos de viver e de se cuidar em situação de rua. Esmeraldo Filho (2010) aposta que a estratégia do CnaR é uma possibilidade de superar algumas dificuldades da atenção básica em relação à pessoa que está em situação de rua. Ferreira (2015) destaca que o atendimento em saúde de pessoas em situação de rua não pode ser algo exclusivo do CnaR.

Com relação à avaliação do serviço do CnaR por seus próprios usuários, pesquisas destacam que é positiva e benéfica (Ferreira, 2015; Ferreira et al., 2016). Entretanto, Abreu (2013) aponta que teve contato com pessoas em situação de rua que ainda não conheciam o serviço. De acordo com o autor, isto pode ocorrer devido aos deslocamentos territoriais que essas pessoas praticam, mas também devido a não construção de vínculo com o serviço. Londero et al. (2014) destacam que alguns serviços ainda apresentam ações encaminhativas e controladoras, pautadas em compreensões morais e normativas. Em contrapartida, Lima (2013) identificou práticas do CnaR realizadas de acordo com os preceitos da redução de danos, e que tanto profissionais entrevistados quanto usuários indicam que tem sido realizada uma prática coerente em saúde visando o cuidado integral e humanizado.

\section{A relação com a cidade}

Para Gomes (2006), pensar pessoas em situação de rua énecessariamente pensar a cidade e o habitar as ruas. $\mathrm{O}$ autor aponta que é possível compreender o habitar as ruas de duas formas: a rua como espaço de interação, encontro e alternativa de vida para algumas pessoas; ou a rua como espaço de abrigo e refúgio, como uma casa. Castiglione (2012) ressalta que se tornar albergado ou se tornar nômade, são estratégias de sobrevivência na cidade. Langa (2012) afirma que, mesmo considerando os fluxos e deslocamentos diários, as pessoas em situação de rua possuem uma rotina e podem estabelecer 
por um curto período de tempo um local fixo para dormir e guardar seus pertences. Esquinca (2013) evidencia que algumas pessoas em situação de rua estabelecem uma relação de apego com o território que ocupam, mas que as vezes precisam se deslocar por diferentes motivos, como necessidades de auxílio social, conflitos com outras pessoas do território e até mesmo devido à ação de terceiros que os afugentam e ameaçam, provocando um deslocamento involuntário.

A circulação, os deslocamentos e a não fixidez em um só território podem estar relacionados ao ordenamento urbano que exige e provoca a mobilidade, através da exclusão dos lugares (Oliveira, 2015). Para Esquinca (2013), há movimentos diferentes em relação aos deslocamentos na cidade, sendo alguns direcionados à periferia, escapando das áreas de revitalização urbana, e outros direcionados à proximidade dos serviços de assistência social, os quais assumem papel relevante na estratégia de sobrevivência (Esquinca, 2013).

O espaço público da cidade configura-se como local de sobrevivência e vivência cotidiana da pessoa em situação de rua (Oliveira, 2011; 2015; Quintão, 2012; Saldanha, 2014; Zwetsch, 2012). Para Quintão (2012), a estrutura urbana é insuficiente para a compreensão dos modos de viver das pessoas em situação de rua, apontando ser necessário planejamento urbano que considere a existência das pessoas que vivem nas ruas, calçadas e becos da cidade.

Para Honorato (2014), existem lugares autorizados e não autorizados para serem habitados por pessoas em situação de rua na cidade. A oferta de casas de acolhimentos e albergues faz parte de uma estratégia de retirada dessas pessoas da rua a fim de manter uma ordem social, justificada pelo incômodo das demais pessoas que não vivem nas ruas, com as pessoas que ali vivem, fomentando e legitimando ações higienistas (Freitas, 2014; Honorato, 2014; Rodrigues, 2009). Para Abib (2014) as intervenções estatais se diferenciam entre as que proporcionam inclusão e as que geram exclusão, mas ambas se constituem como estratégias biopolíticas de controle social.

Pesquisas apontam que algumas pessoas em situação de rua utilizam albergues quando necessitam de segurança, saúde e assistência social, apesar das exigências descontextualizadas e da condição insalubre de alguns deles (Al Alam, 2014; Guedes, 2014). Pessoas em tratamento de saúde devido ao diagnóstico de AIDS, com deficiência física e mulheres, comumente utilizam os albergues como forma de minimizar os riscos e vulnerabilidades da rua (Castiglioni, 2012; Langa, 2012; Rodrigues, 2009). Apesar dos apontamentos críticos em relação ao papel controlador que os albergues podem assumir, para Karam (2015), mesmo com os limites e as regras, esses funcionam como um dispositivo que minimiza os riscos e a vulnerabilidade social.

\section{Os processos de saídas das ruas}

Mattos (2006) destaca a importância de compreender o processo de saída das ruas para o delineamento de políticas públicas direcionada à população de rua, entendendo este processo como uma forma criativa de estabelecer novos modos de viver e enfrentar as dificuldades sociais.

Souza e Araújo (2007) apontam como possibilidade de saída das ruas políticas públicas que auxiliem na construção de projetos de vida, sendo necessário uma rede de apoio e fortalecimento social. Fernandes (2013) destaca o serviço de assistência social de acolhimento em repúblicas para adultos como um potente serviço que resgata autonomia e cidadania, pois nesse espaço a pessoa acolhida tem o direito de ir e vir, conviver com outras pessoas em ambiente residencial, construir seu projeto de vida e reorganizar a questão escolar, profissional e financeira.

Reis (2013) acredita que a recusa de pessoas em situação de rua a saírem desta condição está relacionado ao sentimento de excentricidade existencial e de liberdade que experimentam nas ruas. Quintão (2012) afirma que, para as pessoas que estão na rua circunstancialmente, os equipamentos socioassistenciais são interessantes, porém ainda insatisfatórios; e para os que consideram a rua como morada, a oferta de albergues é infundada, pois não aderem a esse tipo de instituição. Assim, Quintão (2012) destaca algumas pessoas aderem ao processo de saída das ruas, porém outras estão sempre chegando; desse modo, é importante se contemplar essas pessoas no projeto urbanístico, a fim de reconhece-las no território urbano e de seu direito à cidade.

Silva (2015) identificou que, para algumas pessoas em situação de rua, é impossível a saída dessa condição em razão da ausência de apoio comunitário e familiar, bem como de políticas públicas ainda pouco eficazes. Como alternativa os autores apontam a integração em associações de catadores de material reciclável, acesso ao trabalho informal, a vinculação a movimentos sociais (Costa, \& Richetti, 2011; Justo, 2008; Mattos, 2006). 
Para Martins (2016), a mobilização social das pessoas em situação de rua é frágil e inexpressiva. Costa (2013) destaca que são muitos os desafios para a consolidação da organização política visto que há oscilações e rotatividade das pessoas devido à condição de rua. Graziolla (2011) aponta a trajetória do Movimento Nacional de População de Rua (MNPR) como uma das formas de organização política, cooperativa e solidária entre as pessoas em situação de rua.

\section{Considerações finais}

A análise da produção científica da última década - 2006 à 2016 - sobre pessoas em situação de rua evidencia a heterogeneidade dessa população e as múltiplas facetas de viver nas ruas das cidades. As produções analisadas corroboram a pesquisa nacional (Brasil, 2009) que evidência a predominância de pessoas do sexo masculino. As justificativas que ocasionam a situação de rua, entre a pesquisa nacional e as demais, também convergem ao considerar a multifatorialidade desta condição, a predominância de relatos referentes ao uso de álcool e outras drogas, desemprego e fragilidade de vínculos; consideraram também a escolha individual como um possível na vida nas ruas. Alguns estudos identificaram que existem pessoas em situação de rua que se culpam por estar nessa condição, individualizando e reduzindo a si mesmas essa responsabilidade. São necessárias investigações que tenham como foco esse processo de culpabilização para uma análise detalhada do problema e de seus efeitos.

Constatamos que as pesquisas possuem interesse em compreender as razões e os motivos das pessoas viverem nas ruas, bem como os diferentes modos de se relacionarem nas e com as ruas. Estão comprometidas com a desconstrução de estereótipos, estigmas e preconceitos relacionados essas pessoas; apresentam e as descrevem de forma humanizada e objetivam desenvolver um conhecimento que possa contribuir para melhorar de alguma forma suas dificuldades.

As pesquisas apresentam variados temas de interesse em relação às pessoas em situação de rua e algumas aprofundaram a investigação na tentativa de compreender características de subgrupos específicos, como pessoas que fazem uso abusivo de drogas, crianças e jovens nas ruas. Com relação à temática gênero, foram encontradas apenas pesquisas referentes a mulheres em situação de rua, não havendo estudos sobre homens, homossexualidades e travestilidades.
Consideramos necessária a produção de conhecimentos com foco nas temáticas de gênero e sexualidade.

A relação das pessoas em situação de rua com a sociedade foi evidenciada nas pesquisas de dois modos: uma relação de exclusão e preconceito por um lado, ou uma relação construída através de auxílios e ajudas, por outro. Essa dicotomia chama a atenção para a dificuldade de realizar generalizações aos modos de ser, viver e se relacionar das pessoas em situação de rua, sendo necessário considerar singularidades, as diferentes maneiras de existir e de se relacionar.

As pesquisas sobre os discursos midiáticos em relação às pessoas em situação de rua demonstram a forte influência social da mídia que contribui para a construção de uma imagem estigmatizante dessas pessoas como vagabundas, inúteis e criminosas. Contrariando esse discurso, as investigações ressaltaram que muitas pessoas em situação de rua desempenham atividades laborais e se encontram ativas no processo de se reinventarem, resistindo às práticas de exclusão e as dificuldades cotidianas. $\mathrm{O}$ processo de saída das ruas, por sua vez, foi compreendido pelas pesquisas como possibilidade e não como obrigatoriedade, apesar de algumas investigações destacarem a falta de planejamento urbanístico das cidades para acolher essas pessoas.

As pesquisas apontaram preocupação com o acesso das pessoas em situação de rua ao Sistema Único de Saúde (SUS) e ao Sistema Único de Assistência Social (SUAS), evidenciando a necessidade de construção de uma rede intersetorial direcionada a essa população. Destacaram a importância de pensar a intersetorialidade de maneira ampliada, considerando habitação, educação e trabalho, além de ações que esclareçam seus direitos sociais. Evidenciaram assim a necessidade de políticas públicas direcionadas a essa população que considerem as especificidades seus diversos subgrupos.

Verificamos que o interesse em investigar o fenômeno de pessoas em situação de rua tem sido crescente no Brasil. As pesquisas, de modo geral, apresentam-se preocupadas em construir informações e contribuir para a produção de ações a elas direcionadas. Mas apesar disso, evidenciamos que se faz necessário o investimento em mais investigações que produzam conhecimentos e informações contextualizadas, coerentes e humanizadas, bem como indicadores para construção de uma política de direitos a essa população. 


\section{Referências}

Abib, L. T. (2014). Crônicas urbanas: Consultório na rua, população em situação de rua, clínica menor e outras histórias (dissertação). Universidade Federal do Rio Grande, RS, Brasil.

Abreu, D. (2013). Pessoas em situação de rua, uso de drogas e o consultório de rua (dissertação). Universidade Federal de Santa Catarina, Florianópolis, SC, Brasil.

Aguiar, M. J. G. (2014). Moradores de rua na cidade do Guarujá/SP: Condições de vida, saúde, emoções e riscos (dissertação). Universidade Católica de Santos, SP, Brasil.

Al Alam, M. C. L. (2014). População em situação de rua: Território como lugar de trabalho em saúde (dissertação). Universidade Federal de Pelotas, Pelotas, RS, Brasil.

Albuquerque, S. C. (2014). Cuidado em saúde frente às vulnerabilidades: Práticas do consultório na rua (dissertação). Pontifícia Universidade Católica de São Paulo, São Paulo, SP, Brasil.

Alcantara, S. C., Abreu, D. P., \& Farias, A. A. (2015). Pessoas em situação de rua: Das trajetórias de exclusão social aos processos emancipatórios de formação de consciência, identidade e sentimento de pertença. Revista Colombiana de Psicologia, 24(1), 129-143. https://doi.org/10.15446/rcp.v24n1.40659.

Alles, N. L. (2010). Boca de rua: Representações sociais sobre população de rua em um jornal comunitário (dissertação). Universidade Federal do Rio Grande do Sul, Porto Alegre, RS, Brasil.

Almeida Junior, G. P. (2014). Pelas ruas e nas páginas das revistas: Estratégias de construção da representação do morador de rua no discurso jornalístico de Ocas" e VEJA São Paulo (dissertação). Escola Superior de Propaganda e Marketing, São Paulo, SP, Brasil.

Alves, M. E. R. (2013). Vidas privadas em espaços públicos: Uma análise da violência contra a mulher moradora de rua em Fortaleza (dissertação). Universidade Estadual do Ceará, Fortaleza, CE, Brasil.

Argiles, M. S. (2012). População adulta em situação de rua: Da invisibilidade social ao direito a ter direitos (dissertação). Universidade Católica de Pelotas, Pelotas, RS, Brasil.

Borysow, I. C., \& Furtado, J. P. (2013). Acesso e intersetorialidade: O acompanhamento de pessoas em situação de rua com transtorno mental. Physis, 23(1), 33-50. https://ddoi.org/10.1590/S0103-73312013000100003

Brasil. (2009). Ministério do Desenvolvimento Social e Combate à Fome. Rua: Aprendendo a contar: Pesquisa Nacional sobre População em Situação de Rua. Brasília, DF: o autor.

Brêtas, A. C. P., Marcolan, J.F., Rosa, A. S., Fernandes, F. S. L. F., \& Raizer, M.V. (2010). Quem mandou ficar velho e morar na rua?. Revista da Escola de Enfermagem da USP, 44(2), 476-481. https://doi.org/10.1590/S0080-62342010000200033

Bueno, E. M. (2013). Os desafios de envelhecer na rua (dissertação). Pontifícia Universidade Católica de São Paulo, São Paulo, SP, Brasil.

Büll, S. (2010). Histórias de trabalho e outras histórias no trecho (dissertação). Pontifícia Universidade Católica de Campinas, SP, Brasil.

Campos, M. A. R. (2012). Sob o céu da cidade: Representações sociais da população em situação de rua no município de Araguari (dissertação). Universidade Federal de Uberlândia, Uberlândia, MG, Brasil.

Candido, N. A. (2006). Ação da pastoral da Igreja Católica Apostólica Romana face ao direito à inserção social de pessoas em situação de rua (dissertação). Universidade Metodista de São Paulo, São Paulo, SP, Brasil.

Carvalho, G. M. (2015). Análise psicodinâmica do trabalho da população de rua de Manaus (dissertação). Universidade Federal do Amazonas, Manaus, AM, Brasil.

Carvalho, S. M. C. (2014). "Os ditos sem” acesso à saúde da população em situação de rua (dissertação). Universidade Católica de Salvador, Salvador, BA, Brasil.

Castiglioni, M. C. (2012). Poesia concreta em prosa no asfalto: Limites da deficiência no espaço urbano. Interface (Botucatu), 16(43), 1087-1093. https://doi.org/10.1590/S1414-32832012005000053

Cofani, A. (2012). Juventude e consumo de álcool entre jovens de distintos grupos sociais (dissertação). Universidade de São Paulo, São Paulo, SP, Brasil.

Conselho Regional de Psicologia Minas Gerais - CRP-MG. (2015). A Psicologia e a população em situação de rua: Novas propostas, velhos desafios. Belo Horizonte, MG: o autor. 
Costa, A. L. P. (2013). Política municipal de atendimento à população em situação de rua de Fortaleza: Desafios para uma proposta de inclusão (dissertação). Universidade Estadual do Ceará, Fortaleza, CE, Brasil.

Costa, D. B. (2009). Cidadãos e cidadãs em situação de rua: Uma análise de discurso crítica da questão social (tese). Universidade de Brasília. Recuperado de http://repositorio.unb.br/handle/10482/4308

Costa, D. L. R. (2007). A rua em movimento: experiência urbanas e jogos sociais em torno da população de rua (dissertação). Universidade de São Paulo, São Paulo, SP, Brasil.

Costa, L. D. G. (2010). Responsabilidade e desumanização: Representaçães sociais sobre população de rua no Rio de Janeiro (dissertação). Pontifícia Universidade Católica do Rio de Janeiro, Rio de Janeiro, RJ, Brasil.

Costa, J. M., \& Richetti, P. (2011). Reflexões sobre exclusão em tempos de globalização: notas sobre viver nas ruas. Hologramatica, 14(3), 3-15. Recuperado de http://www.cienciared.com.ar/ra/usr/3/468/hologramatica_n14_v3pp3_15.pdf

Costa, L. E., Mesquita, V. M., \& Campos, A. P. (2015). Moradores de rua, quem são eles? Um estudo sobre a população de rua atendida pela Casa da Sopa “Capitão Vendramini" de Três Corações. Revista da Universidade Vale do Rio Verde, 13(2), 285-297. https:/ / doi.org/10.5892/ruvrd.v13i2.2281

Cunha, J. G. (2015). Pessoas em situação de rua e seus cães: Fragmentos de união em histórias de fragmentação (dissertação). Universidade Federal do Espírito Santo, Vitória, ES, Brasil.

Esmeraldo Filho, C. E. (2010). Necessidades de saúde dos moradores de rua: desafios para as políticas sociais do município de Fortaleza-CE (dissertação). Universidade Estadual do Ceará, Fortaleza, CE, Brasil.

Esquinca, M. M. M. (2013). Os deslocamentos territoriais dos adultos moradores de rua nos bairros Sé e República (dissertação). Universidade de São Paulo, São Paulo, SP, Brasil.

Farias, V. C. C. (2007). Possibilidades de inserção/reinserção produtiva dos moradores de rua do município de Porto Alegre (dissertação). Pontifícia Universidade Católica do Rio Grande do Sul, Porto Alegre, RS, Brasil.

Félix-Silva, A. V., Sales, R. C. M., \& Soares, G. P. (2016). Modos de viver e fazer arte de pessoas em situação de rua. Estudos de Psicologia, 21(1), 46-57. https://doi.org/10.5935/1678-4669.20160006

Fernandes, C. N. (2013). Estudo sobre o serviço de acolhimento em república para adultos em situação de rua do município de São Paulo (dissertação). Universidade Federal do ABC, Santo André, SP, Brasil.

Ferreira, A. J. P. (2007). A comunicação presencial de sem-tetos na cidade de São Paulo: A produção e distribuição da Revista Ocas (dissertação). Pontifícia Universidade Católica de São Paulo, São Paulo, SP, Brasil.

Ferreira, C. P. S. (2015). Consultório na Rua em Maceió/AL: O olhar de pessoas em situação de rua (dissertação). Universidade Federal de Juiz de Fora, Juiz de Fora, MG, Brasil.

Ferreira, N. S. A. (2002). As pesquisas denominadas "estado da arte". Educação \& Sociedade, 9(1), 257-272. https:// doi.org/10.1590/S0101-73302002000300013

Ferreira, C. P. S.; Rozendo, C. A. \& Melo, G. B. (2016). Consultório na Rua em uma capital do Nordeste brasileiro: O olhar de pessoas em situação de vulnerabilidade social. Cadernos de Saúde Pública, 32(8), 1-10. https://doi. org/10.1590/0102-311X00070515

Frazão, T. C. J. (2010). O morador de rua e a invisibilidade do sujeito no discurso jornalístico (tese). Universidade de Brasília, Brasília, DF, Brasil.

Freitas, C. J. (2014). "Os indesejáveis": Agentes públicos e a gestão da mobilidade de trecheiros e pessoas em situação de rua (dissertação). Universidade Estadual Paulista, Assis, SP, Brasil.

Galvani, D. (2008). Pessoas em situação de rua na cidade de São Paulo: Itinerários e estratégias na construção de redes sociais e identidades (dissertação). Universidade de São Paulo, São Paulo, SP, Brasil.

Galvani, D. (2015). Circuitos e práticas religiosas nas trajetórias de vida de adultos em situação de rua na cidade de São Paulo (tese). Universidade de São Paulo, São Paulo, SP, Brasil.

Garcia, R. C. M., Calderón, N., \& Ferreira, F. (2012). Consolidação de diretrizes internacionais de manejo de populações caninas em áreas urbanas e proposta de indicadores para seu gerenciamento. Revista Panamericana de Salud Publica, 32(2), 140-144.

Gomes, R. C. M. (2006). Gente-caracol: A cidade contemporânea e o habitar as ruas (dissertação). Universidade Federal do Rio Grande do Sul, Porto Alegre, RS, Brasil. 
Gomes, B. R., \& Adorno, R. C. F. (2011). Tornar-se "noia": Trajetória e sofrimento social nos "usos de crack" no centro de São Paulo. Etnográfica, 15(3), 569-586.

Graziolla, R. (2011). As pessoas em situação de rua em Porto Alegre e seus dramas, tramas e manhas: A cooperação e a solidariedade como forma de humanização (dissertação). Universidade Federal do Rio Grande do Sul, Porto Alegre, RS, Brasil.

Guedes, W. F. (2014). O centro comunitário São Martinho de Lima: Um espaço de vivência para a população em situação de rua em São Paulo (dissertação). Pontifícia Universidade Católica São Paulo, São Paulo, SP, Brasil.

Guimarães, A. G. C. (2010). A religiosidade de moradores de rua da cidade de Belo Horizonte: Uma via de subjetivação (dissertação). Pontifícia Universidade Católica de Minas Gerais, Belo Horizonte, MG, Brasil.

Honorato, B. E. F. (2014). Ordem e subversão nas cidades: Um estudo sobre a população em situação de rua de Belo Horizonte (dissertação). Universidade Federal de Minas Gerais, Belo Horizonte, MG, Brasil.

Justo, M. G. (2008). Vida nas ruas de São Paulo e alternativas possíveis: Um enfoque sócio-ambiental. InterfacEHS, 3(1), 4. Recuperado de http:/ / www.revistas.sp.senac.br/index.php/ITF/article/viewFile/91/116

Karam, B. J. (2015). O egresso prisional em situação de rua no Estado de São Paulo (dissertação). Pontifícia Universidade Católica de São Paulo, São Paulo, SP, Brasil.

Lacerda, T. F. (2012). Bioética e diversidade: Condições de saúde da população adulta em situação de rua no Distrito Federal (dissertação). Universidade de Brasília, Brasília, DF, Brasil.

Langa, E. N. B. (2012). Pessoas que habitam as ruas em Fortaleza nos circuitos da vulnerabilidade e exclusão: Identidades em construção nas trajetórias e percursos (dissertação). Universidade Federal do Ceará, Fortaleza, CE, Brasil.

Lemões, T. (2014). A corporificação do sofrimento e o trânsito entre vítima e algoz: novas reflexões a partir de etnografias com população em situação de rua. Cadernos do Lepaarq, 11(21), 45-61. Recuperado de https://periodicos.ufpel.edu.br/ojs2/index.php/lepaarq/article/view/3155

Lima, H. S. (2013). Consultório na Rua em Goiânia: Atenção a pessoas em situação de rua e em uso de substâncias psicoativas (dissertação). Universidade de Brasília, Brasília, DF, Brasil.

Lisboa, M. S. (2013). Os loucos de rua e as redes de saúde mental: Os desafios do cuidado no território e a armadilha da institucionalização (tese). Pontifícia Universidade Católica de São Paulo, São Paulo, SP, Brasil.

Londero, M. F. P., Ceccim, R. B., \& Bilibio, L. F. S. (2014). Consultório de/na rua: Desafio para um cuidado em verso na saúde. Interface (Botucatu), 18(49), 251-260. https://doi.org/10.1590/1807-57622013.0738

Macerata, I., Soares, J. G. N., \& Ramos, J. F. C. (2014). Apoio como cuidado de territórios existenciais: Atenção Básica e a rua. Interface (Botucatu), 18, 919-930. https://doi.org/10.1590/1807-57622013.0210

Martins, D. R. (2016). Receita pra lavar praça suja: Políticas públicas de saúde mental para a população em situação no Centro de Referência Especializado da Assistência Social da cidade de Leme/SP (dissertação). Universidade Estadual de Campinas, Campinas, SP, Brasil.

Matias, H. J. D. (2013). Sedução e descaminho: Narrativas e identidades de jovens em situação de rua. Psicologia: Reflexão e Crítica, 26(3), 543-551. https://doi.org/10.1590/S0102-79722013000300014

Matraga, M. V. C., \& Araújo-Jorge, T. C., \& Wimmer, G. (2014). O PalhaSUS e a saúde em movimento nas ruas: Relato de um encontro. Interface (Botucatu), 18(suppl 2), 1529-1536. https://doi.org/10.1590/1807-57622013.0373

Mattos, R. M. (2006). Situação de rua e modernidade: A saída das ruas como proxesso de criação de novas formas de vida na atualidade (dissertação). Universidade São Marcos, São Paulo, SP, Brasil.

Mendes, K. T., Ronzani, T. M., \& Paiva, F. S. (no prelo). População em situação de rua, vulnerabilidades e drogas: uma revisão sistemática. Psicologia \& Sociedade.

Mendonça, G. C. (2006). Sentidos subjetivos de moradores de rua frente ao futuro (dissertação). Pontifica Universidade Católica de Campinas, Campinas, SP, Brasil.

Miranda, F. A. (2014). Direito à saúde da população em situação de rua (dissertação). Universidade Católica de Salvador, Salvador, BA, Brasil.

Montiel, J. M.; Bartholomeu, D; Carvalho, L. F \& Pessotto, F. (2015). Avaliação de transtornos da personalidade em moradores de rua. Psicologia, Ciência e Profissão. 35(2), 488-502. https://doi.org/10.1590/1982-370301992013 
Morera, J. A. C. (2013). Crack: Histórias de vida de moradores de rua (dissertação ). Universidade Federal de Santa Catarina, Florianópolis, SC, Brasil.

Moura, Y. G., Silva, E. A., \& Noto, A. R. (2009). Redes sociais no contexto de uso de drogas entre crianças e adolescentes em situação de rua. Psicologia em Pesquisa, 3(1), 31-46.

Moura Junior, J. F. (2012). Reflexões sobre a pobreza a partir da identidade de pessoas em situação de rua de Fortaleza (dissertação). Universidade Federal do Ceará, Fortaleza, CE, Brasil.

Nogueira, F. G. P. (2008). Hóspedes incômodos: estudo sobre moradores de rua no hospital de emergência (dissertação). Pontifícia Universidade Católica do Rio de Janeiro, Rio de Janeiro, RJ, Brasil.

Oliveira, L. M. F. (2012). Circulação e fixação: O dispositivo de gerenciamento dos moradores de rua em São Carlos e a emergência de uma população (dissertação). Universidade Federal de São Carlos, São Carlos, SP, Brasil.

Oliveira, M. M. (2015). “Acham que brotamos das fontes dessa cidade?”Uma etnografia sobre o cotidiano de sobrevivência de pessoas em situação de rua em Natal/RN (dissertação). Universidade Federal do Rio Grande do Norte, Natal, RN, Brasil.

Oliveira, M. R. L. (2011). A rua como espaço para morar: Observações sobre a apropriação dos espaços públicos pelos moradores de rua da cidade de João Pessoa-PB (dissertação). Universidade Federal da Pararaíba, João Pessoa, PB, Brasil.

Oliveira, A. A. S., Trancoso, A. E. R., Bastos, J. A., \& Canuto, L. T. (2015). Metassíntese: Apontamentos para sistematização de revisões amplas e crítica interna à produção científica. Atas CIAIQ2015. Recuperado de https://proceedings.ciaiq.org/index.php/ciaiq2015/article/view/36/34

Quintão, P. R. (2012). Morar na rua: Há projeto possível? (dissertação). Universidade de São Paulo, São Paulo, SP, Brasil.

Reis, D. F. (2013). A cidade do morador de rua e o morador de rua na cidade: Lugar e percepção do ambiente urbano (dissertação). Universidade Federal Fluminense, Niterói, RJ, Brasil.

Reis, M. S. (2014). Centro de referência especializado para população em situação de rua (Centro Pop) de Londrina (PR): Uma análise a partir da perspectiva do usuário (dissertação). Universidade Estadual de Londrina, Londrina, PR, Brasil.

Resende, V.M. (2016). Discursive representation and violation of homeless people's rights: Symbolic violencein Brazilian online journalism. Discourse \& Communication, 10(6), 596-613. https://doi.org/10.1177/1750481316674778

Rodrigues, I. S. (2015). A construção social do morador de rua: O controle simbólico da identidade (dissertação). Universidade Federal de Juiz de Fora, Juiz de Fora, MG, Brasil.

Rodrigues, P. G. (2009). Gênero entre as ruas e a trama institucional: um estudo sobre a vivência de mulheres adolescentes em situação de vulnerabilidade social no centro de São Paulo (dissertação). Universidade Estadual Paulista, Marília, SP, Brasil.

Rosa, A. S., \& Brêtas, A. C. P. (2015). A violência na vida de mulheres em situação de rua na cidade de São Paulo, Brasil. Interface (Botucatu), 19(53), 275-285. https://doi.org/10.1590/1807-57622014.0221

Rosa, A. S.; Secco, M. G., \& Brêtas, A. C. P. (2006). O cuidado em situação de rua: Revendo o significado do processo saúde-doença. Revista Brasileira de Enfermagem, 59(3), 331-336. https://doi.org/10.1590/S0034-71672006000300015

Saldanha, R. M. B. (2014). Dormitório urbano: “Uma problemática social (in)sustentável” (dissertação). Pontifícia Universidade Católica do Rio de Janeiro, Rio de Janeiro, RJ, Brasil.

Sambu, A. (2014). De volta para a casa: Análise de uma política pública voltada à assistência social das pessoas em situação de rua em João Pessoa - Paraíba (dissertação). Universidade Federal de Campina Grande, Campina Grande, PB, Brasil.

Santos, A. A. (2013). O jornal =boca de rua= espaço de possibilidades para pessoas em situação de rua: Uma reflexão discursiva crítica (dissertação). Universidade de Brasília, Brasília, DF, Brasil.

Santos, V. B. (2014). Mulheres em vivência de rua e a integralidade no cuidado em saúde (dissertação). Universidade Federal de Santa Maria, Santa Maria, RS, Brasil.

Serrano, C. E. G. (2013). Homem de rua, homem doente: A população de rua nos discursos do acolhimento institucional público (tese). Universidade de São Paulo, São Paulo, SP, Brasil.

Silva, M. L. L. (2006). Mudanças recentes no mundo do trabalho e o fenômeno população em situação de rua no Brasil 1995-2005 (dissertação). Universidade de Brasília, Brasília, DF, Brasil.

Silva, P. M. F. (2015). Pessoas em situação de rua em Recife: Cidadania através do trabalho como uma alternativa (dissertação). Universidade Federal de Pernambuco, Recife, PE, Brasil. 
Silva, V. M. S. (2014). Pés excluídos: O imaginário religioso da população em situação de rua em João Pessoa-PB (dissertação). Universidade Federal da Paraíba, João Pessoa, PB, Brasil.

Silva, W. N., \& Hüning, S. M. (2015). De morador de rua a criminoso. Athenea Digital, 15(2), 141-165. https://doi. org/10.5565/rev/athenea.1479

Silveira, M. Y. J. (2016). O cuidado à saúde das pessoas em situação de rua: Invisibilidade das ações intersetoriais na cidade de São Carlos - SP (dissertação). Universidade Federal de São Carlos, São Carlos, SP, Brasil.

Souza, E. S., Silva, S. R. V., \& Caricari, A. M. (2007). Rede social e promoção de saúde dos “descartáveis urbanos”. Revista da Escola de Enfermagem da USP, 41(n spe), 810-814. https:// doi.org/ 10.1590/S0080-62342007000500012

Souza, P., \& Araújo, M. C. (2007). Projeto Portal da Inclusão: A experiência dos participantes do abrigo municipal em Maringá - Paraná. Emancipação, 7(2), 181-207.

Souza, W. A. (2015). A assistência social e o trabalho com as pessoas em situação de rua no CREAS: Um campo de intercessão (dissertação). Universidade Estadual Paulista, Assis, SP, Brasil.

Tondin, M. C., Barros Neta, M. A. P., \& Passos, L. A. (2013). Consultório de Rua: Intervenção ao uso de drogas com pessoas em situação de rua. Revista de Educação Pública, 22(49), 485-501.

Varanda, W. (2009). Liminaridade, bebidas alcoólicas e outras drogas: Funções e significados entre moradores de rua (tese). Universidade de São Paulo, São Paulo, SP, Brasil.

Zoltowski, A. P. C., Costa, A. B., Teixeira, M. A. P., \& Koller, S. H. (2014) Qualidade metodológica das revisões sistemáticas em periódicos de psicologia brasileiros. Psicologia: Teoria e Pesquisa, 30(1), 97-104. https://doi.org/10.1590/ S0102-37722014000100012

Zwetsch, B. E. (2012). Limiares urbanos: A necessária precariedade à existência (dissertação). Universidade Federal do Rio Grande do Sul, Porto Alegre, RS, Brasil.

\section{Aline Amaral Sicari}

Mestre em Psicologia Social e Cultura pela Universidade Federal de Santa Catarina (UFSC), Florianópolis - SC. Brasil.

E-mail: alinesicari@hotmail.com

\section{Andrea Vieira Zanella}

Professora Titular da Universidade Federal de Santa Catarina, bolsista em produtividade do CNPq, Florianópolis SC. Brasil.

Endereço para envio de correspondência:

Universidade Federal de Santa Catarina, Centro de Filosofia e Ciências Humanas, Departamento de Psicologia.

UFSC - CFH - Departamento de Psicologia, Trindade, 88010-970 - Florianópolis, SC - Brasil.

Recebido 08/08/2017

Aprovado 25/01/2018

Received 08/08/2017

Approved 01/25/2018

Recibido 08/08/2017

Aceptado 25/01/2018 
Como citar: Sicari, A. A., Zanella, A. V. (2018). Pessoas em situação de rua no Brasil: Revisão sistemática. Psicologia: Ciência e Profissão, 38(4), 662-679. https://doi.org/10.1590/1982-3703003292017

How to cite: Sicari, A. A., Zanella, A. V. (2018). Homeless people in Brazil: A systematic review. Psicologia: Ciência e Profissão, 38(4), 662-679. https:// doi.org/10.1590/1982-3703003292017

Cómo citar: Sicari, A. A., Zanella, A. V. (2018). Personas en situación de calle en Brasil: Revisión sistemática. Psicologia: Ciência e Profissão, 38(4), 662-679. https://doi.org/10.1590/1982-3703003292017 